\title{
Editorial
}

\section{A\&A makes the transition to article numbering}

\author{
C. Bertout ${ }^{1}$, K.S. de Boer ${ }^{2}$, G. Meynet ${ }^{3}$, and M. Walmsley ${ }^{4}$ \\ ${ }^{1}$ Editor-in-Chief, Astronomy \& Astrophysics, Observatoire de Paris, 61 Av. de l'Observatoire, 75014 Paris, France \\ 2 Board of Directors, Astronomy \& Astrophysics, Argelander Institut für Astronomie, Auf dem Hügel 71, 53121 Bonn, Germany \\ Board of Directors, Astronomy \& Astrophysics, Obs. de Genève, 51 Chem. des maillettes, 1290 Sauverny, Switzerland \\ ${ }^{4}$ Letter Editor-in-Chief, Astronomy \& Astrophysics, Osservatorio Astrofisica de Arcetri, Largo E.Fermi 5, 50125 Firenze, Italy
}

Quick dissemination of validated science results is an important aspect of academic publishing. Over the years, the electronic editions of journals have become the primary vehicle for achieving that objective. With the new contract period for publishing A\&A, the preparation of the electronic edition is being optimized for speed of publication.

To streamline the production of the Journal, it has appeared useful to change from the classical "page-numbering system" to a more flexible "article-numbering system". This means that, as of January 2010, articles will be marked according to their class of publication using a roman letter: $\mathrm{L}$ for Letter, A for Article, $\mathrm{E}$ for Editorial, $\mathrm{C}$ for Corrigendum, etc. Furthermore, the articles of each class of publication will be given a number in the volume based on the sequence in which they are ready for publication in that volume of A\&A.

Published manuscripts will thus be numbered in A\&A with a number preceded by a roman letter signifying the publication class. The result is that they will appear in A\&A as, e.g.,
A\&A 512, L1 for the Letter that is published first in Volume 512
A\&A 512, A12 for the twelfth published article in the same volume
A\&A 512, A13 for the thirteenth published article, and so on.

The title page of the published manuscript will provide its full reference, e.g.,

$$
\begin{aligned}
& \text { A\&A 512, L1 (2010) } \\
& \text { A\&A 512, A12 (2010). }
\end{aligned}
$$

The page numbering will be internal to each manuscript, i.e., the first page of each paper will always be numbered as 1 . The page number will be located at the bottom of each page and will take the form "Page $n$ out of $N$ " where $N$ is the total page number of the manuscript and $1 \leq n \leq N$.

Finally, the paper's reference will be repeated as the running title of even-numbered pages while the running title of oddnumbered pages will be left unchanged.

The manuscripts accepted for publication will appear in the currently opened A\&A volume as soon as they are ready to become public. A\&A users will also be able to see on the Journal website how the open volume is being filled up every day with newly published manuscripts. At the closure date of the volume, its contents will be sent to the printer. The printed volumes of A\&A will contain the publications in the sequence E1, E2, etc., L1, L2, L3, etc., A1, A2, A3, etc. However, the A\&A website and the table of contents of each volume will display the published papers in the usual manner, with a table of contents sorted by sections.

Authors do not need to worry about the changes, since everything will be taken care of in the editorial and production process.

Citing an article will be in a format only slightly different from earlier since there will always be a capital letter in front of the last number. For Letter number 4 and article number 12 in Volume 552, the references will be as follows.

Writer, U. V., \& Author, B. C. 2010, A\&A, 552, L4

Author, B. C. 2010, A\&A, 552, A12 